\title{
Vorwort zur 108. Auflage
}

Die chemische Analytik ist nicht nur ein wichtiges Teilgebiet in vielen naturwissenschaftlichen Studiengängen, sie spielt auch eine permanent wichtige Rolle in den Medien, wenn es z.B. um die Bewertung von Fragen der Toxikologie, der Lebensmittelsicherheit, des Umweltschutzes oder der Klimaveränderungen geht.

Den Studenten aller naturwissenschaftlichen Fachrichtungen stehen dazu heute per Mausklick eine Vielzahl von Informationen zur Verfügung, die früher mühsam zusammengesucht werden mussten. Zur kritischen Bewertung und sinnvollen Verarbeitung dieser Informationen ist aber ein grundlegendes Verständnis der fundamentalen Zusammenhänge erforderlich. Dieses Rüstzeug in Form von Daten, Formeln, Beispielen und Erklärungen stellt der „Küster-Thiel“ in aktualisierter und optimierter Form zur Verfügung.

So wurden in der vorliegenden Auflage die Zahlentabellen zugunsten zusätzlicher Übungsbeispiele im Bereich physikalisch-chemischer Berechnungen wie Elektrodenpotenziale, pH-Werte usw. auf das Notwendigste reduziert. Desweiteren wurden in einem neuen Kapitel quantitative chromatographische Methoden wie z. B. High Performance Liquid Chromatography (HPLC) aufgenommen. Dazu notwendige Kalibrierverfahren, Optimierung der Trennleistung sowie Beurteilung und Auswertung von Peakflächen wurden ergänzt und mit Beispielen hinterlegt.

Für vielfältige Anregungen bedanken wir uns sehr herzlich bei unseren Nutzern. Unser besonderer Dank gilt Herrn Dr. Eduard Sorkau, Martin-Luther-Universität Halle-Wittenberg für seine konstruktiven Beiträge und dem Verlag de Gruyter für die konstruktive Zusammenarbeit.

Altenbach im Juli 2016

Ursula Ruland

Alfred Ruland 